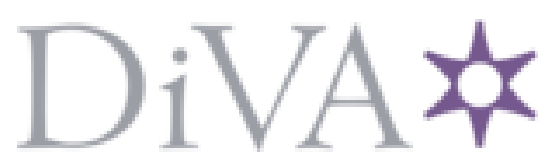

http://www.diva-portal.org

This is the published version of a paper published in Journal of Chemical Physics.

Citation for the original published paper (version of record):

Kieri, E., Holmgren, S., Karlsson, H. (2012)

An adaptive pseudospectral method for wave packet dynamics.

Journal of Chemical Physics, 137: 044111:1-12

http://dx.doi.org/10.1063/1.4737893

Access to the published version may require subscription.

N.B. When citing this work, cite the original published paper.

Copyright (2012) American Institute of Physics. This article may be downloaded for personal use only. Any other use requires prior permission of the author and the American Institute of Physics.

Permanent link to this version:

http://urn.kb.se/resolve?urn=urn:nbn:se:uu:diva- 179058 


\section{Uppsala University}

This is a published version of a paper published in Journal of Chemical Physics.

Citation for the published paper:

Kieri, E., Holmgren, S., Karlsson, H. (2012)

"An adaptive pseudospectral method for wave packet dynamics"

Journal of Chemical Physics, 137(4): 044111:1-12

URL: http://dx.doi.org/10.1063/1.4737893

Access to the published version may require subscription.

Copyright 2012 American Institute of Physics. This article may be downloaded for personal use only. Any other use requires prior permission of the author and the American Institute of Physics.

Permanent link to this version:

http://urn.kb.se/resolve?urn=urn:nbn:se:uu:diva-179058

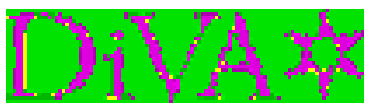

http://uu.diva-portal.org 


\title{
An adaptive pseudospectral method for wave packet dynamics
}

\author{
Emil Kieri, ${ }^{1}$,a) Sverker Holmgren, ${ }^{1}$ and Hans O. Karlsson ${ }^{2}$ \\ ${ }^{1}$ Division of Scientific Computing, Department of Information Technology, Uppsala University, Sweden \\ ${ }^{2}$ Theoretical Chemistry, Department of Chemistry - Angström Laboratory, Uppsala University, Sweden
}

(Received 4 May 2012; accepted 5 July 2012; published online 26 July 2012)

\begin{abstract}
We solve the time-dependent Schrödinger equation for molecular dynamics using a pseudospectral method with global, exponentially decaying, Hagedorn basis functions. The approximation properties of the Hagedorn basis depend strongly on the scaling of the spatial coordinates. Using results from control theory we develop a time-dependent scaling which adaptively matches the basis to the wave packet. The method requires no knowledge of the Hessian of the potential. The viability of the method is demonstrated on a model for the photodissociation of IBr, using a Fourier basis in the bound state and Hagedorn bases in the dissociative states. Using the new approach to adapting the basis we are able to solve the problem with less than half the number of basis functions otherwise necessary. We also present calculations on a two-dimensional model of $\mathrm{CO}_{2}$ where the new method considerably reduces the required number of basis functions compared to the Fourier pseudospectral method. (C) 2012 American Institute of Physics. [http://dx.doi.org/10.1063/1.4737893]
\end{abstract}

\section{INTRODUCTION}

The detailed understanding of the dynamics of chemical reactions is a fundamental challenge in chemical physics. Mathematical models describing dynamic quantum mechanical processes have provided important tools for such understanding for almost one hundred years. Such models are based on the time-dependent Schrödinger equation (TDSE) which can only be solved analytically in very simplified settings. Hence, numerical solution methods are of great importance to provide a deeper theoretical understanding of reaction dynamics and to design and interpret experimental studies. In this paper, we solve the TDSE for molecular dynamics,

$$
\begin{gathered}
\mathrm{i} \hbar \frac{\partial}{\partial t} \Psi=\hat{H} \Psi, \text { where } \\
\hat{H}=-\sum_{j=1}^{d} \frac{\hbar^{2}}{2 m_{j}} \frac{\partial^{2}}{\partial x_{j}^{2}}+V(\mathbf{x}, t),
\end{gathered}
$$

using an expansion of the wave function $\Psi$ in a non-standard time-adaptive basis set consisting of global but exponentially decaying functions. $\mathbf{x}$ denotes the nuclear degrees of freedom. We study the dynamics of a wave packet on a potential energy surface (PES), as well as photo-induced and non-adiabatic coupling of different PES. Common to the problems studied is that the wave packet on a given PES stays localised and that it occupies different parts of the computational domain at different times, i.e., it has bounded, time-dependent support. This opens for spatial adaptivity. We can save computational work if we resolve only the part of space currently occupied by the wave packet, instead of resolving the whole computational domain at all times.

Basis function expansions have been dominant in computational methods for quantum molecular dynamics since

\footnotetext{
a)Electronic mail: emil.kieri@it.uu.se.
}

decades. Well-established examples of such computational methods, using different basis sets, are given in, e.g., Refs. 1-3. We use the basis set due to Hagedorn, ${ }^{4,5}$ which is a generalisation to arbitrary dimensions of complex Hermite functions. A Hagedorn function subject to appropriate evolution of its parameters is the exact solution of the TDSE with a quadratic potential. In Ref. 6, Faou et al. constructed a computational algorithm for the TDSE using a Galerkin approximation on spaces of Hagedorn functions. The algorithm can be related to the time-dependent discrete variable representation (TDDVR) developed by Billing and co-workers. ${ }^{7-9}$ In one spatial dimension, the Hagedorn and TDDVR basis functions are identical up to a variable substitution. However, the Hagedorn basis is more flexible when used for problems with multiple degrees of freedom and provides a promising framework for more challenging quantum dynamics problems. This paper presents a new computational scheme for wave packet dynamics which is based on the algorithm in Ref. 6, but improves the approximation properties of the basis set by introducing an adaptive scaling. By using well-established results from control theory we can match the support of the basis to the wave packet. We also improve the computational complexity for practical computations by using a collocation (pseudospectral) scheme instead of a Galerkin (spectral) approach.

Control theory has been used before to improve numerical methods for differential equations. In Ref. 10, Gustafsson et al. used control theory to design a step size controller for Runge-Kutta methods which is robust with respect to stiffness. In this work, we use control theory for spatial adaptivity through moving meshes. When working with moving meshes one faces two questions: where should the mesh points be moved, and how should they be brought there? Moving mesh methods have been studied within the numerical analysis community. An extensive, recent review is given in Ref. 11. The most well-studied cases are formulated on timeindependent, bounded domains, where the indicator functions 
for where to move the mesh points typically are given through local a posteriori error estimates. In Ref. 12, Pettey and Wyatt presented a moving mesh method for the TDSE. They used the Fourier representation for calculating spatial derivatives and needed a uniform grid. They let the grid spacing be timeindependent and did not move interior grid points. Instead, they added or removed points near the boundaries according to indicators for the support of the solution, given through semiclassical trajectory tracing. Boundary treatment is an omnipresent issue for unbounded domain problems such as the Schrödinger equation. On fixed grids one can either make the domain big enough for the wave function to stay away from the boundary, or use absorbing boundary conditions ${ }^{13,14}$ which dampen outgoing waves. The present method adaptively changes the size and location of the computational domain such that the wave function is negligible by the boundaries.

In one spatial dimension, the Hagedorn functions are complex Gaussians multiplied by Hermite polynomials. The convergence of an approximation using a Hermite basis can be perceived as slow compared to approximation in, e.g., the Fourier basis. ${ }^{15}$ This is a consequence of the Hermite functions being global. While the Fourier basis lives on a finite interval, the Hermite functions form a basis of $L_{2}(\mathbb{R})$. If the basis consists of $K$ functions, the highest resolvable frequency grows as $\mathcal{O}(K)$ for the Fourier basis and as $\mathcal{O}(\sqrt{K})$ for the Hermite basis, as $K \rightarrow \infty$. However, at the same time, the support of the Hermite basis grows as $\mathcal{O}(\sqrt{K}) .{ }^{16}$ This compromise between the resolution of higher frequencies and larger support for the Hermite basis is an intriguing and potentially useful property. Also for functions of finite support, the approximation error can be reduced considerably by introducing a $K$-dependent scaling of the basis. ${ }^{17}$ In Ref. 18, a timedependent scaling of a Hermite basis was used for parabolic equations and the viscous Burgers' equation. In the present paper, we introduce an adaptive scaling which makes the support of the basis follow the support of the wave packet. As noted by Billing and Adhikari, ${ }^{8}$ using the classical equations of motion for propagating the shape parameters of the basis is not in all cases a good choice. By using a basis which is better adapted to the solution one gets better accuracy with fewer basis functions. The number of basis function needed, and thus also the computational complexity and memory requirements, still scales exponentially with the number of dimensions. This is a feature shared with other methods aiming at resolving the wave function. ${ }^{2,7,19}$ However, the savings of being able to use a smaller basis also grows with dimensionality. While still constrained to low-dimensional problems, adaptivity can make larger problems accessible for computation.

In Secs. II and III we review the construction of Hagedorn wave packets and their time evolution. We discuss the Galerkin time propagation used in Ref. 6 and an alternative collocation method. In Sec. IV we discuss the approximation properties of the Hagedorn basis and give motivation to the need for spatial adaptivity. We formulate a control target, i.e., conditions for the desired basis, which in Sec. V is developed into a controller which dynamically adapts the basis to the evolving wave function. We demonstrate the performance of the new method on a photodissociation problem, an IBr model with three PES, in Sec. VI. Coupled PES is a strongly quantum mechanical phenomenon which makes classical propagation of the basis parameters infeasible. By letting the evolution of the solution determine the propagation of basis parameters, this problem is elegantly solved. We also consider propagation on a multi-dimensional PES, a collinear model of the $\mathrm{CO}_{2}$ molecule, in Sec. VII. The spatial adaptivity improves the resolution capabilities of the basis, reducing the error substantially.

\section{HAGEDORN WAVE PACKETS}

A Hagedorn wave packet ${ }^{4,5}$ is an expansion of the wave function on a global basis which diagonalises the Hamiltonian for the quantum mechanical harmonic oscillator. In this section we will review the construction of the Hagedorn basis and the propagation algorithm of Ref. 6 for solving the TDSE for quantum molecular dynamics. We consider the TDSE in semiclassical scaling,

$$
\mathrm{i} \varepsilon \frac{\partial}{\partial t} \Psi=-\sum_{j=1}^{d} \frac{\varepsilon^{2}}{2 m_{j}} \frac{\partial^{2}}{\partial x_{j}^{2}} \Psi+V \Psi,
$$

where the variables have been scaled as $t \rightarrow t / \varepsilon, m \rightarrow m / \varepsilon^{2}$. $\Psi$ is the nuclear wave function and $\varepsilon$ is a free scaling parameter, conventionally chosen as the reciprocal square root of a characteristic nuclear mass. This scaling is convenient since it scales the involved quantities to similar orders of magnitude. It also allows us to classify problems, since the value of $\varepsilon$ gives an indication to how close to classical mechanics the system is. Note that we do not treat the method semiclassically, considering convergence in the classical limit $\varepsilon \rightarrow 0$. Instead, we consider fixed, finite $\varepsilon$ and a collocation method for fully quantum mechanical problems. We are thus dependent on resolving the high-frequency oscillations of the wave function. Let $\varphi_{\mathbf{k}}$ denote the $d$-dimensional basis functions, where the index vector $\mathbf{k}=\left(k_{1}, \ldots, k_{d}\right)^{T}, k_{j}=0$, $1, \ldots$ We also introduce the unit vectors $\mathbf{e}_{j}$ with $\left(\mathbf{e}_{j}\right)_{i}=\delta_{i j}$. The Hagedorn basis functions are characterised by the parameters $\varepsilon \in \mathbb{R} ; \mathbf{q}, \mathbf{p} \in \mathbb{R}^{d} ; Q, P \in \mathbb{C}^{d \times d}$, and are defined by

$$
\begin{gathered}
\varphi_{\mathbf{0}}(\mathbf{x})=(\pi \varepsilon)^{-\frac{d}{4}}(\operatorname{det} Q)^{-\frac{1}{2}} \\
\times \exp \left(\frac{\mathrm{i}}{2 \varepsilon}(\mathbf{x}-\mathbf{q})^{T} P Q^{-1}(\mathbf{x}-\mathbf{q})+\frac{\mathrm{i}}{\varepsilon} \mathbf{p}^{T}(\mathbf{x}-\mathbf{q})\right) \\
\hat{\mathcal{R}}_{j} \varphi_{\mathbf{k}}=\sqrt{k_{j}+1} \varphi_{\mathbf{k}+\mathbf{e}_{j}}, \\
\hat{\mathcal{L}}_{j} \varphi_{\mathbf{k}}=\sqrt{k_{j}} \varphi_{\mathbf{k}-\mathbf{e}_{j}}
\end{gathered}
$$

with the raising and lowering operators

$$
\hat{\mathcal{R}}=\frac{\mathrm{i}}{\sqrt{2 \varepsilon}}\left(P^{*}(\hat{\mathbf{x}}-\mathbf{q})-Q^{*}(\hat{\mathbf{p}}-\mathbf{p})\right),
$$




$$
\hat{\mathcal{L}}=-\frac{\mathrm{i}}{\sqrt{2 \varepsilon}}\left(P^{T}(\hat{\mathbf{x}}-\mathbf{q})-Q^{T}(\hat{\mathbf{p}}-\mathbf{p})\right) .
$$

Here, $\hat{\mathbf{p}}=-i \varepsilon \nabla$ is the momentum operator, and $\hat{\mathcal{L}}$ is the adjoint operator of $\hat{\mathcal{R}}$. Equations (5)-(8) can be combined into the two-step recurrence relation

$$
Q\left(\sqrt{k_{j}+1} \varphi_{\mathbf{k}+\mathbf{e}_{j}}\right)_{j=1}^{d}=\sqrt{\frac{2}{\varepsilon}}(\mathbf{x}-\mathbf{q}) \varphi_{\mathbf{k}}-\bar{Q}\left(\sqrt{k_{j}} \varphi_{\mathbf{k}-\mathbf{e}_{j}}\right)_{j=1}^{d},
$$

which is convenient since it does not involve differentiation. $(\cdot)_{j=1}^{d}$ denotes a $d$-dimensional vector. The parameters $Q$ and $P$ are required to satisfy the compatibility relations

$$
\begin{gathered}
Q^{T} P-P^{T} Q=0, \\
Q^{*} P-P^{*} Q=2 \mathrm{i} I .
\end{gathered}
$$

A wave packet is expressed as a linear combination of the basis functions $\varphi_{\mathbf{k}}$ multiplied by a common phase factor,

$$
\Psi(\mathbf{x}, t)=e^{\mathrm{i} S(t) / \varepsilon} \sum_{\mathbf{k}} c_{\mathbf{k}}(t) \varphi_{\mathbf{k}}(\mathbf{x}) .
$$

We split the potential in a local harmonic approximation and a remainder,

$$
V(\mathbf{x})=V(\mathbf{q})+(\mathbf{x}-\mathbf{q})^{T} \tilde{V}_{1}+\frac{1}{2}(\mathbf{x}-\mathbf{q})^{T} \tilde{V}_{2}(\mathbf{x}-\mathbf{q})+W_{\mathbf{q}}(\mathbf{x}) .
$$

In Ref. $6 \tilde{V}_{1}=\nabla V(\mathbf{q})$ and $\tilde{V}_{2}=\nabla^{2} V(\mathbf{q})$, the gradient vector and Hessian matrix of $V(\mathbf{x})$ evaluated at $\mathbf{q}$, were chosen. Let $M=\operatorname{diag}\left(m_{j}\right)$. With the expansion (12) of the wave function, the TDSE can be transformed to the following system of ordinary differential equations:

$$
\begin{gathered}
\dot{\mathbf{q}}=M^{-1} \mathbf{p}, \\
\dot{\mathbf{p}}=-\tilde{V}_{1}, \\
\dot{Q}=M^{-1} P, \\
\dot{P}=-\tilde{V}_{2} Q, \\
\dot{S}=\frac{1}{2} \mathbf{p}^{T} M^{-1} \mathbf{p}-V(\mathbf{q}), \\
\mathrm{i} \varepsilon \dot{\mathbf{c}}=F \mathbf{c}, \quad F_{\mathbf{j} \mathbf{k}}=\left\langle\varphi_{\mathbf{j}}\left|W_{\mathbf{q}}\right| \varphi_{\mathbf{k}}\right\rangle .
\end{gathered}
$$

If $\tilde{V}_{1}$ and $\tilde{V}_{2}$ are chosen as the gradient and Hessian of the potential, respectively, $W_{\mathbf{q}} \equiv 0$ and the propagation is exact with time-independent $\mathbf{c}$ if the potential is harmonic. We refer to this potential splitting as semiclassical propagation of (14)-(17). If we do not have a harmonic potential we get a Galerkin-type approximation on the time-dependent Hilbert space $\mathcal{H}$ spanned by the Hagedorn basis functions. This approximation approaches the exact solution when $K \rightarrow \infty$, since the Hagedorn basis is complete. ${ }^{5}$ Also, since $F$ is a Hermitian matrix, exponential time propagation of (19) as in Ref. 6 is norm conserving. The matrix elements $\left\langle\varphi_{\mathbf{j}}\left|W_{\mathbf{q}}\right| \varphi_{\mathbf{k}}\right\rangle$ can in general not be evaluated analytically, but are calculated with Gauss-Hermite quadrature. The Gauss-Hermite quadrature rule using $K_{q}$ nodes is exact for integrands consisting of an exponential kernel and a polynomial part of order up to $2 K_{q}-1 . K_{q} \sim K$ is thus enough for accurate evaluation of the integrals. Note that the matrix elements with the highest order basis functions are normally multiplied with very small expansion coefficients. Loss of accuracy in quadrature there typically has small impact on the solution as a whole. To be safe one might want to use a few more quadrature nodes than basis functions. Throughout this paper we will use $K_{q}=K+3$.

\section{TIME STEPPING}

In this section we consider how to make the time stepping more efficient. As in Ref. 6, we integrate the system (14)-(18) with the second order accurate Störmer-Verlet scheme, splitting the Hamiltonian in its kinetic and potential parts. The coefficients $\mathbf{c}$ were in Ref. 6 propagated using exponential integration with a Krylov subspace method. Direct application of an exponential integrator on the Galerkin equation (19) is expensive. In each time step we need to evaluate the $K \times K$ matrix $F$. Each element in $F$ is defined as an integral, which is evaluated with Gauss-Hermite quadrature using $K_{q} \sim K$ points. The computational effort in each time step then scales as $\mathcal{O}\left(K^{3}\right)$. For practical computations this quickly becomes prohibitive and an approach for reducing the computational cost is needed. We tackle this problem by using a collocation approach instead of the Galerkin method. In each time step we then need to

- evaluate the wave function in $K_{q}$ quadrature points,

- apply the action of $W_{\mathbf{q}}$, and

- project back to the Hagedorn space.

In the Hagedorn space, the wave function $\Psi$ is given as a linear combination of $K$ basis functions. Evaluating $\Psi$ in $K_{q}$ quadrature points,

$$
\Psi\left(\gamma_{j}, t_{n}\right)=\sum_{k=0}^{K-1} c_{k} \varphi_{k}\left(\gamma_{j}\right), \quad j=1, \ldots, K_{q},
$$

requires $\mathcal{O}\left(K^{2}\right)$ operations. We do not need to consider the phase factor $e^{\mathrm{i} S / \varepsilon}$ since it will cancel out in the projection step. In coordinate space the potential energy operator is diagonal. Thus applying the action of $W_{\mathbf{q}}$,

$$
\Psi\left(\gamma_{j}, t_{n+1}\right)=e^{\frac{\Delta t}{\mathrm{i} \varepsilon} W\left(\gamma_{j}\right)} \Psi\left(\gamma_{j}, t_{n}\right), \quad j=1, \ldots, K_{q},
$$

is done in $\mathcal{O}(K)$ operations. Projecting back to the Hagedorn space requires the evaluation of $K$ integrals,

$$
c_{k}=\left\langle\varphi_{k} \mid \Psi\left(\cdot, t_{n+1}\right)\right\rangle,
$$

at the total cost of $\mathcal{O}\left(K^{2}\right)$ operations. Hence, using collocation instead of Galerkin reduces the computational complexity from cubic to quadratic in the number of basis functions.

If we take the limit $\Delta t \rightarrow 0$ we reobtain (19), i.e., the collocation method is consistent with the Galerkin approximation. However, as a consequence of the orthogonal projection $\ell_{2} \rightarrow \mathcal{H}$ in (22) the method is no longer norm conserving, and it is formally only first order accurate in $\Delta t$. We now argue that this is of minor importance. 


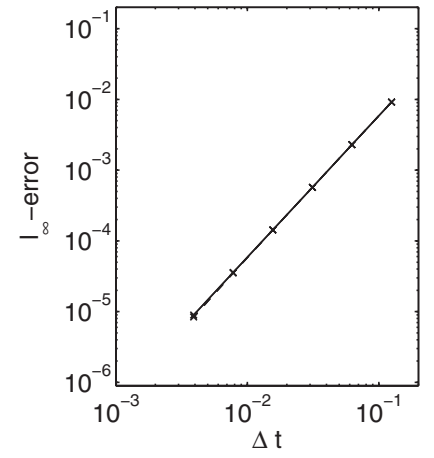

(a)

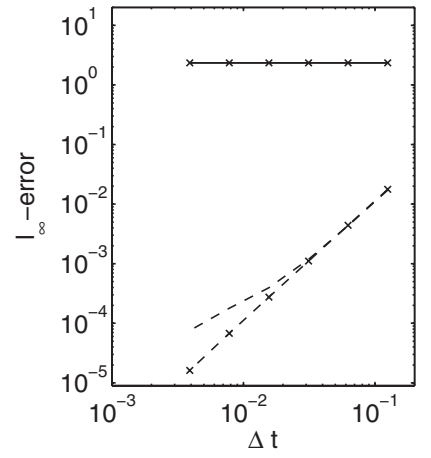

(b)
FIG. 1. Error as function of the time step for Galerkin (crosses) and collocation (plain lines). Errors compared to a reference solution within the Hagedorn space are shown with dashed lines, and errors compared to a spatially well-resolved reference are shown with solid lines. The TDSE is integrated up to $t=1$ with 64 basis functions in (a), and to $t=5$ with 8 basis functions in (b).

When propagating the coefficients $\mathbf{c}$ given an approximation space $\mathcal{H}$, we make two different errors: the $\mathcal{O}\left(\Delta t^{2}\right)$ splitting error between the kinetic and potential parts of the Hamiltonian, and the $\mathcal{O}(\Delta t)$ projection error. However, the projection error is non-zero only when the solution extends outside the approximation space. Then the error induced by the truncation of the basis will be dominant anyway, since the time discretisation error is a result of inability to represent the solution well in the approximation space. As an example, consider the one-dimensional (1D) TDSE for the torsion potential, $V=1-\cos x$. We use $\varepsilon=0.1$, and a Gaussian initial condition with parameters $q=1, p=0, Q=1$, and $P=\mathrm{i}$. If we integrate up to $t=1$ using 64 Hagedorn basis functions, the solution will be well resolved. Consequently, the projection error will be negligible and collocation and Galerkin will yield essentially identical solutions, as can be seen in Figure 1(a). However, if we integrate up to $t=5$ with only 8 basis functions we will not be able to resolve the solution, and we will get an $\mathcal{O}(\Delta t)$ projection error from collocation. If we compare to a reference solution calculated with small time steps but the same number of basis functions, i.e., study the accuracy of time-integration only, the Galerkin approach gives much smaller error. It will be second order accurate, compared to first order for collocation. But if we compare to a spatially well-resolved solution, we see that this does not transfer to the full partial differential equation problem. In Figure 1(b) we see how the spatial error dominates. We thus argue that collocation is more sensible computationally, though Galerkin is more elegant mathematically.

The reference solution was calculated with short-iterative Lanczos (SIL) time integration with a time step $\Delta t=2^{-10}$ $\approx 0.00098$, and a well-resolved Fourier basis consisting of 128 basis functions on the interval $[-\pi, \pi)$.

\section{ADAPTIVE SCALING OF THE BASIS SET}

We now turn to the approximation properties of the Hagedorn basis and present new tools for optimising them. The $K_{q}$ optimal interpolation nodes, given a one-dimensional
Gauss-Hermite basis set $\left\{\varphi_{k}\right\}_{k=0}^{K-1}$, are the zeros of $\varphi_{K_{q}}$, i.e., the nodes for the corresponding Gauss-Hermite quadrature rule. For a motivation, as well as a description of the relation between Gauss quadrature and interpolation, we refer to Chap. 4.7 of Ref. 20. We denote the quadrature nodes by $\gamma_{1}<\gamma_{2}<\cdots<\gamma_{K_{q}}$. These nodes give us an indication of our ability to resolve the wave function. The node spacing determines the highest frequency that can be resolved, and the extension of the node set determines the support of the basis. If the wave function is not small at the most peripheral nodes the representation will suffer from oscillations because of the Runge phenomenon. The node distribution for GaussHermite quadrature is not far from uniform on the interval $\left[\gamma_{1}, \gamma_{K_{q}}\right]$, but slightly denser close to the midpoint. As was mentioned in the Introduction, the node spacing $\Delta x$ decreases as $\mathcal{O}(1 / \sqrt{K})$ as the number of basis functions $K$ increases, while the distance between the most peripheral nodes $\gamma_{1}$ and $\gamma_{K_{q}}$ increases as $\mathcal{O}(\sqrt{K})$. Here, $\Delta x$ is an approximate measure since the node spacing is not fully uniform. The ability to resolve high-frequency oscillations within the support for a wave packet thus increases as $\mathcal{O}(\sqrt{K})$, compared to $\mathcal{O}(K)$ for the Fourier and Chebyshev bases. The Fourier and Chebyshev bases are however defined on a finite interval $I \subset \mathbb{R}$. If the function to be approximated is defined on an infinite domain one has to truncate it, introducing a domain truncation error. For the method to converge, $I$ must approach $\mathbb{R}$ as $K$ $\rightarrow \infty$. If we let $|I|$ grow as $\mathcal{O}(\sqrt{K})$ the highest resolvable frequency would also grow as $\mathcal{O}(\sqrt{K})$, just as for Hermite functions. For a more detailed discussion of different approximation techniques for infinite domains, see, e.g., Chap. 17 of Ref. 16.

Billing and Adhikari noted ${ }^{8}$ that propagation of the width parameter of the Gauss-Hermite basis determined by a harmonic approximation was not optimal in all cases. In particular, they were concerned by the basis occasionally getting very narrow support, resulting in limited ability to resolve peripheral parts of the wave packet. Their solution was a fixed-width treatment where $\tilde{V}_{2}$ was chosen such that the width parameter stayed constant, similar to the frozen Gaussians of Heller. ${ }^{21}$ Here, we present a time-dependent scaling which adapts the basis to the wave packet.

Any wave packet will have bounded support. If the spatial variables are scaled, the support of the basis functions can be adapted to match the wave packet. In Ref. 17, Tang illustrated how the convergence of interpolation can be sped up by scaling the basis functions. By choosing a $K$ dependent scaling factor, we can weight the importance of resolving high frequencies contra large domains. As long as $\Delta x$ $\rightarrow 0$ and $\left(\gamma_{K_{q}}-\gamma_{1}\right) \rightarrow \infty$, the approximation will converge as $K \rightarrow \infty$. The importance of choosing an appropriate scaling factor is illustrated in Figure 2, where a function is projected to two different Hagedorn spaces. The spaces differ by a factor two in the spread parameter $Q$, resulting in drastic difference in ability of representing the function in question. In real simulations with finite-precision arithmetics, the condition of formal asymptotic convergence as $K \rightarrow \infty$ can be relaxed. We may instead scale the basis functions so that $|\Psi(x)|$ $\leq \delta$ outside $\left[\gamma_{1}, \gamma_{K_{q}}\right]$ for some small $\delta$ which determines the 


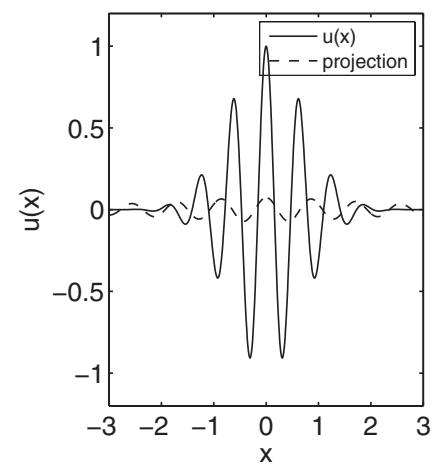

(a)

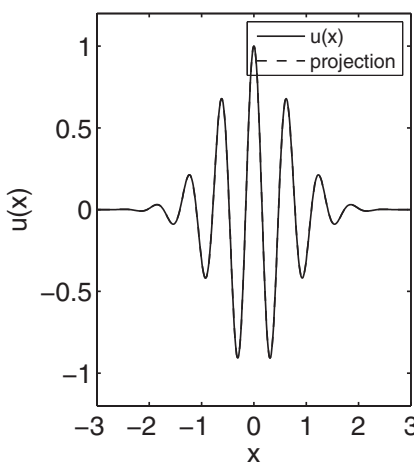

(b)
FIG. 2. Projection of $u(x)=\cos (10 x) e^{-x^{2}}$ to two different Hagedorn spaces, both with $K=32$ basis functions. The figures show $u(x)$ with solid lines and its projection with dashed lines. In both cases, $\varepsilon=1, q=p=0$, $P=\mathrm{i} / Q$. In (a), $Q=1$ and in (b), $Q=0.5$. The $\ell_{\infty}$-errors are 0.93 and 1.8 $\times 10^{-5}$, respectively. In (b), the two lines are indistinguishable.

domain truncation error. The philosophy of this approach is similar to that of domain truncation dependent methods, such as the Fourier method. If the domain truncation is chosen independently of the number of basis functions in the Fourier method there will be a non-vanishing domain truncation error, destroying formal convergence. The support of the Hagedorn basis functions is determined by the parameter $Q$ and the semiclassical scaling parameter $\varepsilon$. The variance of the one-dimensional basis function $\varphi_{k}$ is $(2 k+1)(\varepsilon / 2) Q Q^{*}$, and we use $Q Q^{*}$ to control the support of the basis set.

The unscaled Gauss-Hermite nodes $\tilde{\gamma}_{k}$ are the zeros of the Hermite polynomial of order $K_{q}$, and can be calculated as the eigenvalues of a tridiagonal matrix. ${ }^{22}$ Let $\tilde{\gamma}_{\mathbf{k}}=\left(\tilde{\gamma}_{k_{1}}, \tilde{\gamma}_{k_{2}}, \ldots, \tilde{\gamma}_{k_{d}}\right)^{T}$. Then the scaled and translated nodes corresponding to a Hagedorn wave packet are given by

$$
\boldsymbol{\gamma}_{\mathbf{k}}=\mathbf{q}+\sqrt{\varepsilon Q Q^{*}} \tilde{\boldsymbol{\gamma}}_{\mathbf{k}} .
$$

The square root of a symmetric positive definite matrix is defined through its eigendecomposition,

$$
\sqrt{Q Q^{*}}=U \sqrt{\Lambda} U^{T}
$$

where $U$ is an orthogonal matrix and $\Lambda=U^{T} Q Q^{*} U$ is diagonal. For simplicity we will restrict $Q$ and $P$ to be diagonal, i.e., we will not skew or rotate the grid. The Hagedorn basis will still be a complete basis of $L_{2}\left(\mathbb{R}^{d}\right)$. We want $|\Psi(\mathbf{x})| \leq \delta$ everywhere outside the convex hull of $\left\{\boldsymbol{\gamma}_{\mathbf{k}}\right\}$. Keeping track of the smallest set fulfilling this property is straight-forward in one dimension, then we only need to track its two boundary points. In multiple dimensions the task is nontrivial. We settle with tracking its boundary points along a number of rays originating from $\mathbf{q}$, extending in the directions of the positive and negative coordinate axes. These boundary points, indexed by $i$, can be expressed as

$$
\mathbf{x}_{\delta}^{(i)}=\mathbf{q}+\hat{\mathbf{x}}_{\delta}^{(i)} r_{\delta}^{(i)}
$$

where $\hat{\mathbf{x}}_{\delta}^{(i)}$ are time-independent unit vectors and $r_{\delta}^{(i)}$ are scalar. If $\delta$ is very small, $\nabla \Psi(\mathbf{x})$ will be small close to $\mathbf{x}_{\delta}^{(i)}$, and the determination of $r_{\delta}^{(i)}$ will be ill-conditioned. We therefore choose a moderately small $\delta$, e.g., $10^{-3}$, and take ad- vantage of the exponential asymptotic decay of the wave function. If the wave function is approximately Gaussian, $\left|\Psi\left(\mathbf{q}+\hat{\mathbf{x}}_{\delta}^{(i)} \eta r_{\delta}^{(i)}\right)\right| \approx \delta^{\eta}$, where $\eta$ can be chosen arbitrarily big without loss of stability. The condition for validity of this approximation is that at $r \geq r_{\delta}^{(i)}$ the polynomial part of the wave function should have negligible influence compared to the exponential part, and can be treated as a constant. There will then exist quadrature nodes beyond $r_{\delta}^{(i)}$ along each ray. We generate the value of the wave function at all $\boldsymbol{\gamma}_{\mathbf{k}}$, they will be needed again when propagating the expansion coefficients c. Along each ray, we use the last quadrature node where $\left|\Psi\left(\boldsymbol{\gamma}_{\mathbf{k}}\right)\right|>\delta$ as approximation for $\mathbf{x}_{\delta}^{(i)}$. We then determine the desired shape of the grid for negligible cost. Let $\boldsymbol{\gamma}_{\mathbf{k}_{i}}$ denote the Gauss quadrature node which is the most peripheral in direction $\hat{\mathbf{x}}_{\delta}^{(i)}$ and centred in all other directions. The desired value of $Q Q^{*}$, which we call the control signal, reads

$$
R=Q Q^{*} \text { such that }\left|\Psi\left(\boldsymbol{\gamma}_{\mathbf{k}_{i}}\right)\right| \approx \delta^{\eta} \text {. }
$$

Equivalently, we have the control target $\boldsymbol{\gamma}_{\mathbf{k}_{i}}=\mathbf{q}+\hat{\mathbf{x}}_{\delta}^{(i)} \eta r_{\delta}^{(i)}$. Since $Q$ and $P$ are diagonal it is sufficient to treat each dimension, and sign, separately. If we consider only the coordinate direction which $\hat{\mathbf{x}}_{\delta}^{(i)}$ is aligned along, the control signal can be expressed as

$$
R_{i}=\frac{\left(\eta r_{\delta}^{(i)}\right)^{2}}{\varepsilon \tilde{\gamma}_{K_{q}}^{2}}
$$

For systems with several PES, we scale $\delta$ by the $\ell_{2}$-norm of the wave function on each PES.

\section{THE CONTROLLER}

In Ref. 6, $Q$ was propagated according to the local harmonic approximation of the potential. The splitting (13) was made according to the Taylor expansion so that $\tilde{V}_{1}=\nabla V(\mathbf{q})$ and $\tilde{V}_{2}=\nabla^{2} V(\mathbf{q})$. By using a different splitting of the potential we can control the value of $Q Q^{*}$, and thus the support of the basis functions. The matrix $\tilde{V}_{2}$ can be treated as a free parameter, which we will require to be real and diagonal. An alternative splitting will no longer yield the exact solution for the harmonic oscillator with a truncated Hermite expansion, but we do not lose convergence for general potentials as long as $\Delta x \rightarrow 0$ and $\gamma_{K_{q}} \rightarrow \infty$ as $K \rightarrow \infty$. By dynamically controlling the support of the basis we put the effort on approximating the wave packet in its support, giving a more accurate representation. Another advantage of this splitting is that we no longer need the Hessian of the potential, which normally is expensive to calculate.

We now consider the problem of how to choose $\tilde{V}_{2}$ in order to give $Q Q^{*}$ the desired value. This can be achieved using standard tools from control theory. ${ }^{23}$ We reformulate the Eqs. (16) and (17) for $Q$ and $P$ as

$$
\begin{aligned}
& \frac{d}{d t} Q Q^{*}=M^{-1} P Q^{*}+Q P^{*} M^{-1}, \\
& \frac{d}{d t} P Q^{*}=-\tilde{V}_{2} Q Q^{*}+P P^{*} M^{-1},
\end{aligned}
$$




$$
\begin{aligned}
\frac{d}{d t} Q P^{*} & =M^{-1} P P^{*}-Q Q^{*} \tilde{V}_{2}^{T}, \\
\frac{d}{d t} P P^{*} & =-\tilde{V}_{2} Q P^{*}-P Q^{*} \tilde{V}_{2}^{T} .
\end{aligned}
$$

By the compatibility relations (10) and (11), $\operatorname{Im}\left(P Q^{*}\right)=I$ at all times. We let $X_{2}=\operatorname{Re}\left(P Q^{*}\right)$ and use the fact that all involved matrices are diagonal and therefore commute and are symmetric. The system (28)-(31) can then be rewritten as

$$
\begin{gathered}
\frac{d}{d t} Q Q^{*}=2 M^{-1} X_{2}, \\
\frac{d}{d t} X_{2}=-\tilde{V}_{2} Q Q^{*}+P P^{*} M^{-1}, \\
\frac{d}{d t} P P^{*}=-2 \tilde{V}_{2} X_{2},
\end{gathered}
$$

where all terms are real. $Q Q^{*}, P P^{*}$, and $M$ are positive definite. The conditions for steady-state are

$$
\left\{\begin{array}{l}
P P^{*}=\tilde{V}_{2} Q Q^{*} M, \\
X_{2}=0, \\
\frac{d}{d t} \tilde{V}_{2}=0,
\end{array}\right.
$$

and the second time-derivative of $Q Q^{*}$ is

$$
\frac{d^{2}}{d t^{2}}\left(Q Q^{*}\right)=2 M^{-1} P P^{*} M^{-1}-2 M^{-1} \tilde{V}_{2} Q Q^{*} .
$$

We now consider the dynamical system (32)-(34) as a control system with output signal $Q Q^{*}$, input signal $\tilde{V}_{2}$, and control signal $R(t)$, where $R$ is a diagonal $d \times d$-matrix. The objective is to choose the input signal $\tilde{V}_{2}$ such that the output signal $Q Q^{*}$ stays close to the control signal $R$. We first make an exact linearisation of the control system by choosing

$$
\begin{gathered}
\tilde{V}_{2}=P P^{*} M^{-1}\left(Q Q^{*}\right)^{-1}-\frac{1}{2} M G\left(Q Q^{*}\right)^{-1}, \\
G=G\left(Q Q^{*}, X_{2}, R\right) .
\end{gathered}
$$

$Q Q^{*}$ is positive definite and therefore invertible. This choice of potential splitting yields

$$
\frac{d^{2}}{d t^{2}}\left(Q Q^{*}\right)=G
$$

if $G$ is real and diagonal. Equation (39) is then the well-known double integrator, which can be controlled to arbitrary accuracy by a proportional-derivative (PD) controller,

$$
G=-\alpha\left(Q Q^{*}-R\right)-\beta 2 M^{-1} X_{2} .
$$

Here, $\alpha$ and $\beta$ are free, positive parameters. The first term is proportional to the deviation of $Q Q^{*}$ from the control signal, and the second term is proportional to the time derivative of $Q Q^{*}$. The performance of the controller is determined by the poles of the Laplace transformed control system (39) and (40),

$$
\begin{aligned}
\mathcal{L}\left\{Q Q^{*}\right\}(s)= & \frac{\alpha \mathcal{L}\{R\}(s)}{s^{2}+\beta s+\alpha} \\
& +\frac{\left.s Q Q^{*}\right|_{t=0}+\left.\beta Q Q^{*}\right|_{t=0}+\left.\frac{d}{d t} Q Q^{*}\right|_{t=0}}{s^{2}+\beta s+\alpha} .
\end{aligned}
$$

By choosing the parameters $\alpha$ and $\beta$ we can place the poles of the system arbitrarily. Pole placement is a standard procedure for controller design. ${ }^{23}$ The performance of a control system is conventionally measured through its step response, i.e., how the output signal reacts to a unit discontinuity in the control signal. The more negative the real parts of the poles, the faster the response. The angle to the real axis determines how oscillatory the response is. If the poles have non-zero imaginary parts there will be overshoot in the step response, and the larger the angle, the more overshoot. By placing the poles far enough out in the left half-plane we can get an arbitrarily fast, arbitrarily well-damped step response. The price is increased stiffness in the ordinary differential equation (17). Thus, the larger $\alpha$ and $\beta$ we choose the smaller time steps we need to take, but typically it is enough to keep them in a regime where accuracy requirements on the solution limit the time step. Steps in the control signal may occur if a hump emerges in the periphery of the wave function, or if a hump drops below $|\Psi(\mathbf{x})|=\delta$. Such steps may result in substantial discontinuities in $\tilde{V}_{2}$, which can impair the stability of the method. It is therefore convenient to put a cap on $|G|$. Since the adaptive scheme is formulated as a double integrator, noise in the control signal is smoothed out. The method is robust to discontinuities, and can increase the accuracy of the spatial approximation substantially.

It is important to note that the present methodology considers a fully quantum mechanical description of the molecular system. It is intended, and will only work, for resolved computations. If important high frequency features of the wave function are not resolved, the expansion coefficients $c_{\mathbf{k}}$ for the highest order basis functions will get significant moduli due to aliasing error. We will then suffer from the Runge phenomenon. This is analogous to the Fourier method, where we need the solution to be essentially zero close to the boundaries. If it is not, the periodic boundary conditions will induce error. In the present approach, the adaptivity will in this case add fuel to the fire. It will include the features caused by the Runge phenomenon in the effective computational domain by stretching the basis, making it even less able to resolve high frequencies. If one cannot afford to resolve the high-frequency content of the wave function one should go for semiclassical methods using uncoupled basis functions. ${ }^{5,24}$

To illustrate the new methodology, we apply it to the 1D torsion potential problem discussed in Sec. III. Using $K=32$ basis functions, the time step $\Delta t=2^{-10}, \alpha=25$, $\beta=10, \delta=10^{-3}$, and $\eta=1.5$, we reduce the pointwise error by an order of magnitude to $3.9 \times 10^{-5}$, compared to $8.2 \times 10^{-4}$ with semiclassical propagation of (16) and (17). This supports our proposition that semiclassical evolution of the basis is not necessarily optimal for a collocation scheme. In Figure 3 we show the time evolution of the uncertainty 


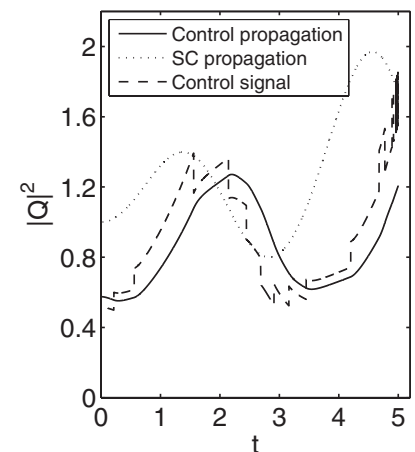

(a)

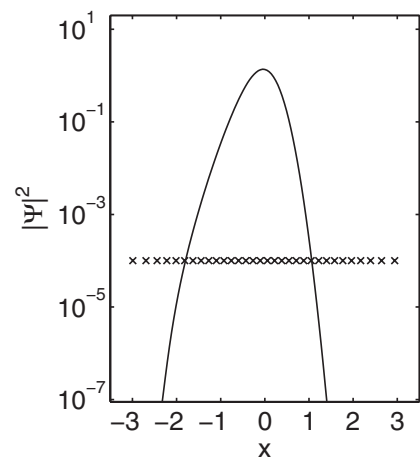

(b)
FIG. 3. (a) The time evolution of the uncertainty parameter $Q Q^{*}$. The solid line indicates control-based propagation and the dotted semiclassical propagation. The dashed line indicates the control signal. (b) A lin-log plot of the squared modulus of the wave function at time $t=5$. The crosses show the location of the collocation points in the control scheme.

parameter $Q$, as well as the Gauss point distribution. Note how the wave function decays rapidly close to the peripheral Gauss points, and is negligible outside the support of the basis. The jaggedness of the control signal is a consequence of the crude approximation of $x_{\delta}$. One can make it smoother by applying linear interpolation or a few steps of bisection on the interval $\left(\gamma_{k}, \gamma_{k+1}\right)$ where the last sign change of $|\Psi(x)|-\delta$ occurs. This would however be of disputable benefit, judging from the smoothness of the output signal $Q Q^{*}$. This amount of noise in the control signal may appear as disturbing from a numerical or physical standpoint, but it is a normal situation in the engineering applications for which the PD controller was developed.

\section{PHOTODISSOCIATION OF IBR}

We now move on to a more realistic problem. We solve the TDSE (1) for the IBr molecule with the three electronic states $X^{1} \Sigma_{0}^{+}, B^{3} \Pi_{0}^{+}$and $Y_{0}^{+}$, as has been done previously in, e.g., Ref. 25. A schematic overview of the molecular system is given in Figure 4. The first vibrational eigenstate $\phi_{0}^{(1)}$ of

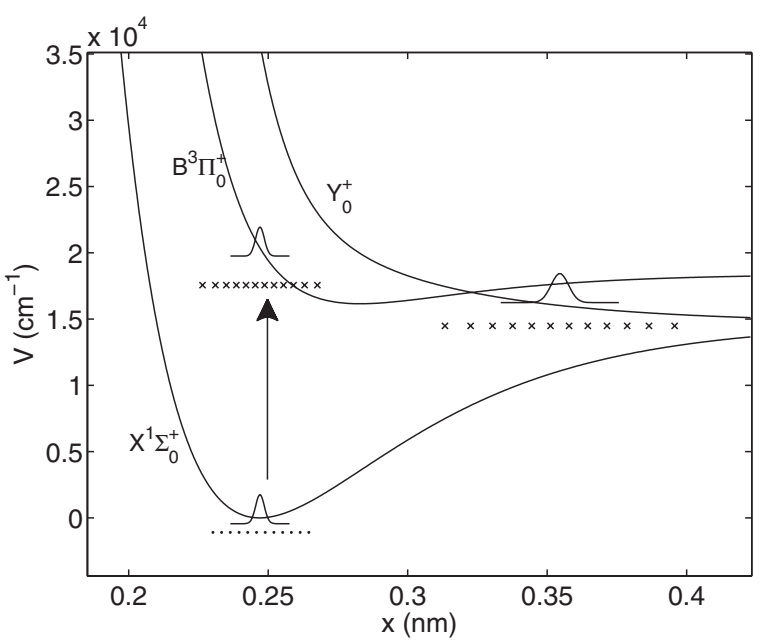

FIG. 4. The PES of the IBr molecule with sketched wave packets and grids. The equidistant Fourier grid of the $X^{1} \Sigma_{0}^{+}$state stays fixed, while the GaussHermite grids in the two excited states follow the wave packets. the ground electronic state $X^{1} \Sigma_{0}^{+}$is taken as initial condition. The $X^{1} \Sigma_{0}^{+}$and $B^{3} \Pi_{0}^{+}$states are coupled by a laser pulse $f(t)$, and the $B^{3} \Pi_{0}^{+}$and $Y_{0}^{+}$states have a non-adiabatic coupling $V_{c}$. The full Hamiltonian of the three-state system then reads

$$
\hat{H}=\left(\begin{array}{ccc}
\hat{H}_{1} & \mu f(t) & 0 \\
\mu f(t) & \hat{H}_{2} & V_{c} \\
0 & V_{c} & \hat{H}_{3}
\end{array}\right) .
$$

The local Hamiltonians are defined by

$$
\hat{H}_{i}=-\frac{\hbar^{2}}{2 m} \frac{\partial^{2}}{\partial x^{2}}+V_{i}(x), \quad i=1,2,3,
$$

where $m=49.0317$ a.u. is the reduced mass of the molecule and $V_{i}(x)$ are the PES. The potentials and pulse parameters are adopted from Refs. 26 and 25, respectively. The coupling term $f(t)=g(t) \cos (\omega t)$ models a laser pulse with envelope function

$$
g(t)=E_{0} e^{-\frac{\left(t-t_{0}\right)^{2}}{2 \sigma^{2}}} .
$$

The dipole moment $\mu$ is taken to be constant, and the pulse parameters are $\lambda=2 \pi c / \omega=500 \mathrm{~nm}, \mu E_{0}=219 \mathrm{~cm}^{-1}$, $t_{0}=60 \mathrm{fs}$, and $\sigma=50 /(2 \sqrt{2 \ln 2})$ fs. The non-adiabatic coupling $V_{c}=150 \mathrm{~cm}^{-1}$. A system of this type poses several computational difficulties:

- The excited states are dissociative. The wave packets thus move over extended distances, and a fixed-grid treatment leads to inefficient use of grid points. For long time propagation, fixed grids require the use of absorbing boundary conditions.

- We want the bases on the respective PES to be able to evolve independently. Implementing the coupling between the states for non-coinciding time-dependent grids is non-trivial.

- The coupling is a quantum phenomenon which occurs during an extended period of time. Evolving the basis according to classical mechanics would give a basis which is poorly adapted to the solution.

Simulation of molecular systems with several PES using Hagedorn wave packets has previously been considered in Ref. 27. In the method proposed there one common set of parameters $\{q, p, Q, P, S\}$, propagated according to one, guiding, PES was used for the wave packets on all surfaces. Each PES had its own set of expansion coefficients $\mathbf{c}$, and the non-diagonal part of the potential, the coupling of the surfaces, was treated together with its non-quadratic remainder. This approach may have difficulties if the wave packets on different PES move away from each other. A large number of basis functions would then be needed in order to accurately represent the wave packets on all surfaces. We propose a different approach using a coupled solver, which uses a pseudospectral method with a Fourier basis in space and Strang splitting ${ }^{28,29}$ in time for the bound $X^{1} \Sigma_{0}^{+}$state, and Hagedorn wave packets for the two dissociative excited states $B^{3} \Pi_{0}^{+}$and $Y_{0}^{+}$. We use one set of parameters per PES. The wave function $\Psi=\left(\begin{array}{lll}\psi_{1} & \psi_{2} & \psi_{3}\end{array}\right)^{T}$ has three components, one for each electronic state. Since the ground electronic state $X^{1} \Sigma_{0}^{+}$is 
bound, the corresponding component of the solution will stay localised. We can therefore without difficulty represent it on a fixed grid, and using a Fourier representation allows us to take advantage of the fast transform when calculating its spatial derivatives. The $B^{3} \Pi_{0}^{+}$and $Y_{0}^{+}$states are however dissociative. The support of the solution on those states is subject to significant variation over time. We would therefore need a lot of grid points for an accurate fixed-grid representation. Using Hagedorn representations for the excited states allows us to let the grids follow the time evolution of the solution.

\section{A. Coupling of the electronic states}

We treat $\psi_{1}$ and the coupling between the states with Strang splitting between the kinetic and potential parts of the Hamiltonian. Since the coupling is time-dependent we use a Magnus expansion. ${ }^{30} \psi_{2}$ and $\psi_{3}$ are handled with a Hagedorn solver. The components are therefore represented on different grids. $\psi_{1}$ is represented on a uniform grid with periodic boundary conditions, $\psi_{2}$ and $\psi_{3}$ are represented by their parameters $q, p, Q, P$, and $S$, and the coefficients $\mathbf{c}$, as described in Sec. II. A Hagedorn representation is associated to a grid of Gauss-Hermite points used for quadrature which follows its movements. The Hamiltonian is split as

$$
\hat{H}=\hat{H}_{P}+\hat{H}_{H} \text {, with }
$$

$$
\hat{H}_{P}=\left(\begin{array}{ccc}
\hat{H}_{1} & \mu f(t) & 0 \\
\mu f(t) & 0 & V_{c} \\
0 & V_{c} & 0
\end{array}\right), \quad \hat{H}_{H}=\left(\begin{array}{ccc}
0 & 0 & 0 \\
0 & \hat{H}_{2} & 0 \\
0 & 0 & \hat{H}_{3}
\end{array}\right) .
$$

The time stepping is made with Strang splitting of the pseudospectral and Hagedorn parts of the Hamiltonian, so that

$$
\Psi^{n+1}=e^{-\mathrm{i} \frac{\Delta t}{2} \hat{H}_{P} / \hbar} e^{-\mathrm{i} \Delta t \hat{H}_{H} / \hbar} e^{-\mathrm{i} \frac{\Delta t}{2} \hat{H}_{P} / \hbar} \Psi^{n} .
$$

Before each time step for the pseudospectral part we need to generate representations of $\psi_{2}$ and $\psi_{3}$ on the uniform grid. Then a time step is made with Strang splitting of a Magnus expansion of $\hat{H}_{P}$. Since the Hagedorn method and Strang splitting are both second order, we use a second order truncation of the Magnus expansion of $\hat{H}_{P}$, which is given in Ref. 31 as

$$
\begin{gathered}
\tilde{H}_{P}=\Delta t\left(\begin{array}{ccc}
\hat{H}_{1} & C_{0} & 0 \\
C_{0} & 0 & V_{c} \\
0 & V_{c} & 0
\end{array}\right), \text { where } \\
C_{0}=\frac{1}{\Delta t} \int_{0}^{\Delta t} \mu f\left(t_{n}+\tau\right) d \tau .
\end{gathered}
$$

The ground electronic state is fully handled with the Fourier pseudospectral method. After the half time step with the Fourier method, the Hagedorn representations of $\psi_{2}$ and $\psi_{3}$ have to be updated according to the transfer between the states. Let

$$
\Delta \psi_{i}=\psi_{i}^{n+\frac{1}{4}}-\psi_{i}^{n}, \quad i=2,3 .
$$

This is then projected back to the Hagedorn spaces via

$$
c_{k, i}^{n+\frac{1}{4}}=c_{k, i}^{n}+\left\langle e^{\mathrm{i} S / \varepsilon} \varphi_{k, i} \mid \Delta \psi_{i}\right\rangle, \quad i=2,3 .
$$

We project the difference $\Delta \psi_{i}$ instead of the whole excited part of the wave function since we want to be able to propagate the Hagedorn wave packet out of the computational domain of the pseudospectral scheme. We interpolate $\Delta \psi_{i}$ to the local grid of state $i$ with cubic splines, and evaluate the integral in (51) using Gauss-Hermite quadrature. After updating the coefficients $c_{k, i}$, we are ready to make a time step with the Hagedorn method. Note that transfer between the electronic states is confined to certain parts of the domain, namely close to where $B^{3} \Pi_{0}^{+}$and $Y_{0}^{+}$cross, and where the separation of $X^{1} \Sigma_{0}^{+}$and $B^{3} \Pi_{0}^{+}$matches the frequency of the laser pulse. There is therefore no need to include the full computational domain in the coupling scheme, we only need to consider the parts where the couplings are active.

\section{B. Determination of parameters for the Hagedorn wave packets}

When probability is excited to a PES for the first time, we need to set the parameters of a new wave packet. Spawning of Hagedorn wave packets has previously been considered in Ref. 32. Given a pointwise defined wave function $\psi$, we set the wave packet parameters according to

$$
\begin{gathered}
q=\frac{\langle\psi|x| \psi\rangle}{\langle\psi \mid \psi\rangle}, \\
p=\frac{\langle\psi|\hat{p}| \psi\rangle}{\langle\psi \mid \psi\rangle}, \\
Q=\frac{\eta r_{\delta}}{\sqrt{\varepsilon} \tilde{\gamma}_{K_{q}}}, \\
P=\frac{\mathrm{i}}{Q} \\
S=0 .
\end{gathered}
$$

The classical coordinate and momentum are set as their expectation values. $Q$ is set so that the most peripheral Gauss point $\gamma_{K_{q}}=q+\eta r_{\delta}$, and $P$ so that the system (28)-(31) starts in steady-state. Since we are doing this with $\psi$ represented on a uniform grid, spatial derivatives are conveniently calculated with a Fourier pseudospectral method. Taking the classical action $S$ to be 0 is no restriction, the initial phase factor will be included in the coefficients $\mathbf{c}$.

The duration of the laser pulse cannot be considered as short compared to the vibrational motion of the nuclei. Transfer between the electronic states is localised in space, but what is excited early in the simulation will have moved considerably by the time the pulse is turned off. The same localisation in space but long duration holds for the non-adiabatic coupling between the two excited states, where the time of transfer is related to the time needed for $\psi_{2}$ to cross the area of coupling. This poses a challenge in the Hagedorn basis. The wave functions on the excited states, $\psi_{2}$ and $\psi_{3}$, get their 


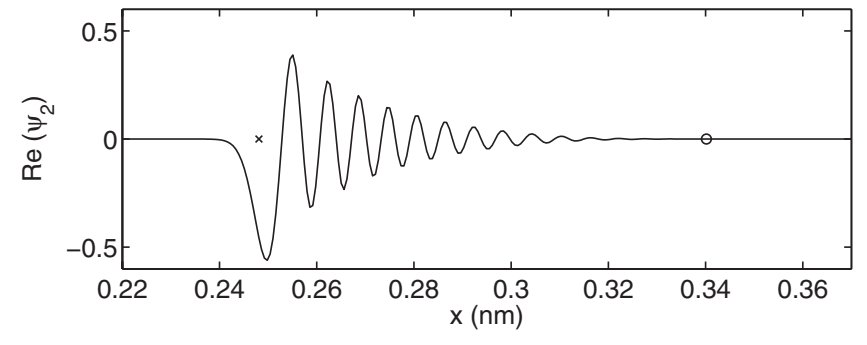

FIG. 5. The real part of $\psi_{2}$ at time $t_{0}$, when the laser pulse peaks. The cross marks the coordinate of strongest coupling with the ground electronic state. The circle marks where the classical coordinate $q$ would have been located if no control were used.

classical coordinates $q$ in the parameterisation which occurs during the first time step where probability is excited. If we let this coordinate follow its classical trajectory as previously it will follow the front of the wave packet, not its centre. We will then spend half of the resolution capabilities of the basis on an interval where the wave function is negligible. Figure 5 shows the wave function $\psi_{2}$ at the time of the peak of the laser pulse. On the first excited state the molecule dissociates. The wave packet moves towards larger $x$-values while more probability is excited around the same coordinate. The approximation properties of the Hagedorn basis would be better if $q$ followed the centre of the wave packet. We achieve this using the same control approach which is used for $Q$.

The time evolution of $q$ is governed by a double integrator, just as for $Q$. Putting the Eq. (14) and (15) together, we get

$$
\ddot{q}=-\frac{1}{m} \tilde{V}_{1} .
$$

The midpoint of the wave packet can be calculated as

$$
\langle x\rangle=\langle\psi|x| \psi\rangle=q+\frac{\sqrt{2 \varepsilon}}{\sum_{k=0}^{K-1} c_{k}^{*} c_{k}} \operatorname{Re}\left(Q \sum_{k=1}^{K-1} c_{k}^{*} c_{k-1} \sqrt{k}\right) .
$$

$\tilde{V}_{2}$ is chosen as before, and in a similar manner we choose

$$
\tilde{V}_{1}=m\left(\alpha(q-\langle x\rangle)+\beta \frac{p}{m}\right) \text {. }
$$

We can then control $q$ to arbitrary accuracy as well, thus being in control of both the position and the spread of the wave packet. We will use the same $\alpha$ and $\beta$ as for $\tilde{V}_{2}$, but one is free to make a different choice.

\section{Computational results}

We integrate the system up to the time $T=120 \mathrm{fs}$ with the new method, taking time steps of length $\Delta t=0.1 \mathrm{fs}$. For the ground electronic state and the photo-induced coupling we consider the spatial interval $x \in[0.20,0.35] \mathrm{nm}$, and for the non-adiabatic coupling $x \in[0.22,0.40] \mathrm{nm}$. The spatial step size is $\Delta x=0.3 / 512 \mathrm{~nm}$. On the excited states we use Hagedorn bases with 256 basis functions per PES. For comparison we compute a reference solution on $x \in[0.2,0.5) \mathrm{nm}$ using a Fourier basis with 512 basis functions per PES. We integrate it in time with SIL, using a fourth order Magnus expansion of the evolution operator, ${ }^{31}$ and the same time step $\Delta t$. We use

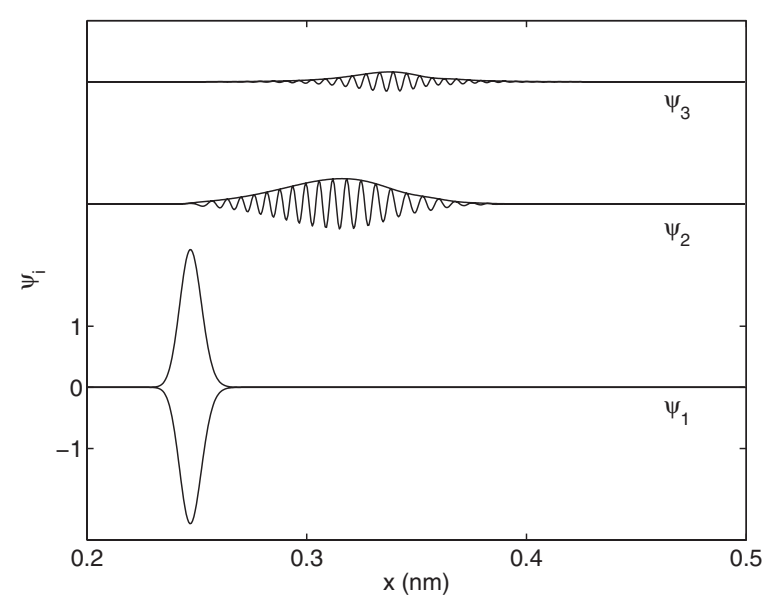

FIG. 6. The solution, real parts and moduli, at the three potential energy surfaces at time $T=120 \mathrm{fs}$.

the control parameters $\alpha=16, \beta=8, \delta=10^{-3}, \eta=2$, and put the cap $G_{\max }=2.5$ on $|G|$.

The solution at $T=120 \mathrm{fs}$ is shown in Figure 6 . The error is then $2.1 \times 10^{-3}$ in the maximum norm given by

$$
\|\Psi\|_{\ell_{\infty}}=\max _{i} \max _{j}\left|\psi_{i}\left(x_{j}\right)\right|,
$$

compared to the reference solution. $x_{j}$ are the nodes of the Fourier grid, the error is measured over the full domain of support of the wave function. The maximum error occurs for $\psi_{1}$, which is expected since most of the probability is there. If we scale by the $\ell_{2}$-norm of the solution, or population, on each state, i.e.,

$$
\|\psi\|=\max _{j} \frac{\left|\psi\left(x_{j}\right)\right|}{\|\psi\|_{\ell_{2}}}
$$

the error is $2.3 \times 10^{-3}, 4.4 \times 10^{-3}$, and $6.0 \times 10^{-3}$ for $\psi_{1}$, $\psi_{2}$ and $\psi_{3}$, respectively.

On the second excited state $Y_{0}^{+}$, a hump eventually appears by the front of the wave function $\psi_{3} . x_{\delta}$ at that front is the rightmost coordinate where $\left|\psi_{3}(x)\right|=\delta\left\|\psi_{3}\right\|_{\ell_{2}}$. As more probability is excited to $Y_{0}^{+},\left\|\psi_{3}\right\|_{\ell_{2}}$ grows and the hump will sink below the limit $\delta\left\|\psi_{3}\right\|_{\ell_{2}}$. There will then be a discontinuity in $x_{\delta}$, and hence also in the control signal. The controller responds with a smooth transition with limited overshoot, as can be seen in Figure 7.

\section{PROPAGATION IN TWO DIMENSIONS}

To show the applicability of the new method to multidimensional problems we apply it to a two-dimensional anharmonic PES. We use the collinear model of an excited electronic state of $\mathrm{CO}_{2}$ presented in Refs. 33 and 34, expressed in the symmetric and asymmetric stretch coordinates $(s, a)$. If $R_{1}$ and $R_{2}$ are the $\mathrm{CO}$ bond distances and $m_{\mathrm{O}}$ and $m_{\mathrm{C}}$ the atomic masses, $s=\left(R_{1}+R_{2}\right) / 2$ and $a=\left(R_{1}-R_{2}\right) /$ $\left(2+m_{\mathrm{C}} / m_{\mathrm{O}}\right)$. The potential is symmetric with respect to the line $a=0$, and has a saddle point at $(s, a)=(2.41,0)$. It has two dissociation channels corresponding to $\mathrm{O}+\mathrm{CO}$ and $\mathrm{OC}+\mathrm{O}$. The PES is illustrated in Figure 8. The masses corresponding to the chosen degrees of freedom are $m_{s}=2 m_{\mathrm{O}}$ and 


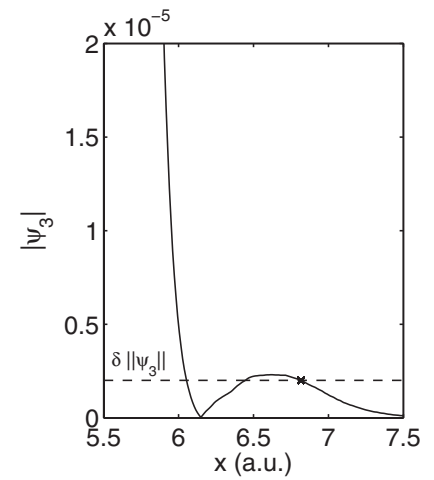

(a)

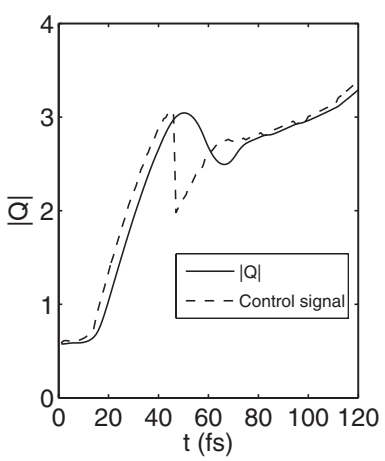

(b)
FIG. 7. (a) The front of $\psi_{3}$ in modulus at time $45 \mathrm{fs}$. The dashed line indicates $\delta\left\|\psi_{3}\right\|_{\ell_{2}}$, and the cross $x_{\delta}$. As $\left\|\psi_{3}\right\|_{\ell_{2}}$ grows, the dashed line will rise above the top of the hump, and $x_{\delta}$ will encounter a discontinuity. The response to this is shown in (b). The dashed line shows the control signal for $|Q|$, and the solid line its actual value.

$m_{a}=m_{\mathrm{C}}\left(1+m_{\mathrm{C}} /\left(2 m_{\mathrm{O}}\right)\right)$. The Hamiltonian operator then reads

$$
\hat{H}=-\frac{\hbar^{2}}{2 m_{s}} \frac{\partial^{2}}{\partial s^{2}}-\frac{\hbar^{2}}{2 m_{a}} \frac{\partial^{2}}{\partial a^{2}}+V(s, a) .
$$

As initial state we use the vibrational ground state of the ground electronic state (sudden approximation). As model for the ground state PES we use a harmonic oscillator ${ }^{34}$ with $\hbar \omega_{s}=1388.3 \mathrm{~cm}^{-1}, \hbar \omega_{a}=2349.0 \mathrm{~cm}^{-1}$. Its vibrational ground state is a Gaussian with centrepoint $(s, a)$ $=\left(s_{0}, 0\right), s_{0}=2.20$ a.u., situated at the bottom of the well. After transfer to the excited state, where the simulation starts, it will be slightly dislocated from the dissociation channels as shown in Figure 8. The initial condition then reads

$$
\psi_{0}=\sqrt{\frac{\sqrt{m_{s} m_{a} \omega_{s} \omega_{a}}}{\pi \hbar}} \exp \left(-\frac{m_{s} \omega_{s}}{2 \hbar}\left(s-s_{0}\right)^{2}-\frac{m_{a} \omega_{a}}{2 \hbar} a^{2}\right) .
$$

We integrate up to the time $T=15 \mathrm{fs}$, and for the Fourier-SIL reference solution we truncate the domain to $(s, a) \in[1.9,3.5)$ $\times[-1.2,1.2)$. We use 256 Fourier basis functions per coordinate direction, and take time steps of $2.42 \times 10^{-3}$ fs for both the Fourier and Hagedorn solutions. Errors are calculated in the $\ell_{\infty}$-norm over the Fourier grid points, covering the full do-

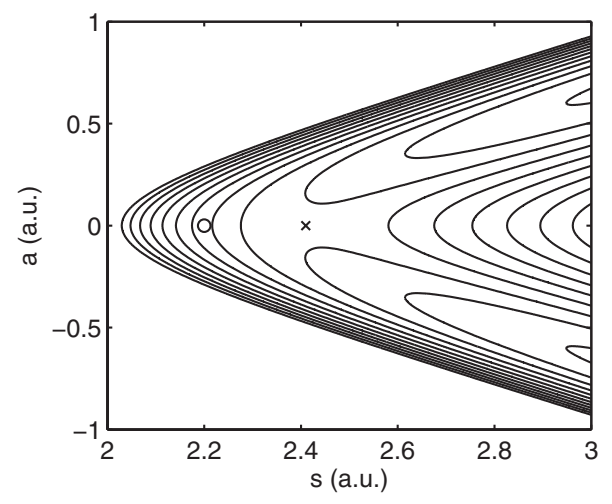

FIG. 8. Level curves for the excited state PES of $\mathrm{CO}_{2}$. The location of the saddle point is indicated by a cross, and the centrepoint of the initial Gaussian wave packet by a ring. The level curves are separated by 0.015 hartree.

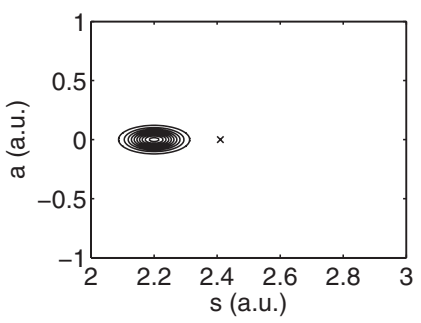

(a)

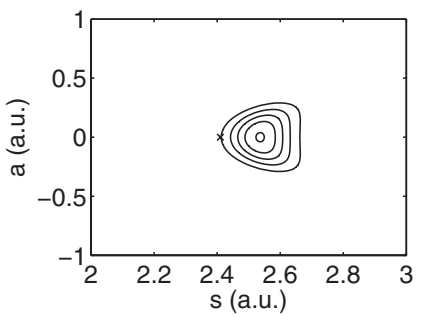

(c)

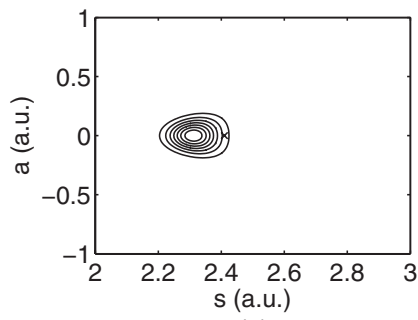

(b)

(d)

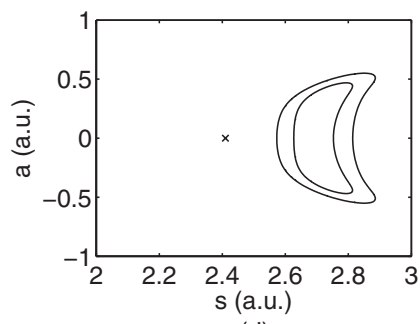

FIG. 9. Time evolution of the wave function. The modulus of the wave function, $|\Psi(s, a)|$, is plotted with a separation of 1 between the level curves. The cross indicates the location of the saddle point in the potential. The wave function is plotted at times $t=$ (a) $0 \mathrm{fs}$, (b) $5 \mathrm{fs}$, (c) $10 \mathrm{fs}$, and (d) $15 \mathrm{fs}$.

main of support of the wave function. The control parameters used are $\delta=10^{-3}, \eta=1.5, \alpha=25$, and $\beta=10$.

The time evolution of the wave function is shown in Figure 9. Initially, the wave packet starts moving towards growing $s$. Its centrepoint crosses the saddle point of the potential at around $t=7 \mathrm{fs}$. It then starts spreading in the asymmetric stretch direction. Semiclassical propagation of $Q$ overdoes this spreading so that we get difficulties resolving the high-frequency oscillations of the wave packet. When using the new method, the growth of $\left|Q_{2}\right|$, and hence the spread of the collocation points, stays more modest, as does the aliasing error. At $t=11 \mathrm{fs}$, the difference in error is between three and four orders of magnitude. However, as the simulation proceeds, the wave packet gets more and more delocalised, and increasingly difficult to resolve. As a result of this, also the error for the control-based propagation starts to grow rapidly around $t=12 \mathrm{fs}$. At the simulation end-time, $t=15 \mathrm{fs}$, the

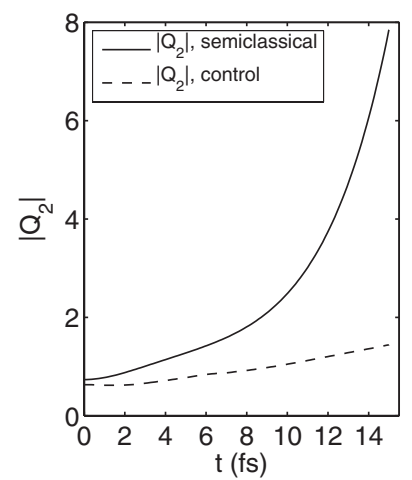

(a)

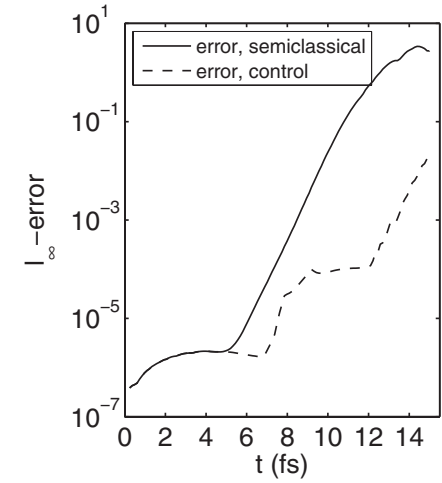

(b)
FIG. 10. (a) The modulus of $Q_{2}$, which determines the spread of the collocation points along the asymmetric stretch coordinate axis, plotted against time. Semiclassical propagation of $Q$ and $P$ is shown with solid lines, and controlbased propagation with dashed lines. (b) The time evolution of the maximum pointwise error for the two propagation schemes. 


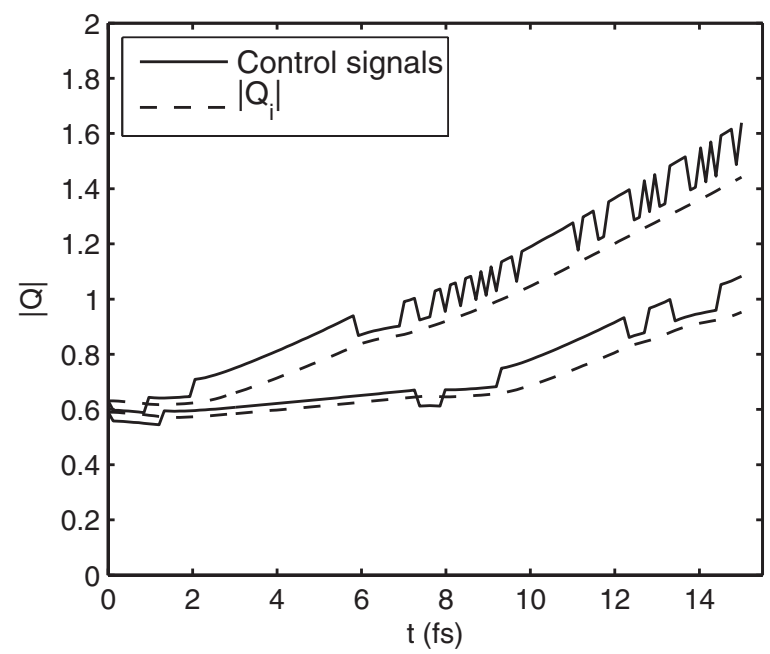

FIG. 11. The performance of the controller. $\left|Q_{1}\right|$ (below) and $\left|Q_{2}\right|$ (above) are plotted against time. The square root of the control signal is shown with solid lines, and the actual parameter values with dashed lines.

error is about 0.020 . In order to proceed further one would have to increase the number of basis functions, and eventually also introduce a mechanism to split the wave packet into two. The time evolution of $\left|Q_{2}\right|$ and the pointwise error is shown in Figure 10. The performance of the controller itself is illustrated in Figure 11. In Figure 12 we illustrate the benefits of a time-dependent basis. The control-based Hagedorn computation with $32^{2}$ basis functions is compared at time $t=10 \mathrm{fs}$ to a computation in a fixed grid Fourier basis on $(s, a) \in[1.9$, $3.0) \times[-0.8,0.8)$, with a polynomial complex absorbing potential (CAP) in $(s, a) \in[2.8,3.0) \times[-0.8,0.8)$, by the right boundary. The potential with CAP is given by

$\tilde{V}(s, a)=V(s, a)-10 \mathrm{i}\left(\frac{s-2.8}{0.2}\right)^{6}, \quad s \in[2.8,3.0)$.

In order to get the $\ell_{\infty}$-error to the same order as for the Hagedorn solution we need 52 Fourier basis functions per coordinate direction. To calculate the pointwise error, a reference solution is calculated on $(s, a) \in[1.9,3.2) \times[-0.8,0.8)$, where

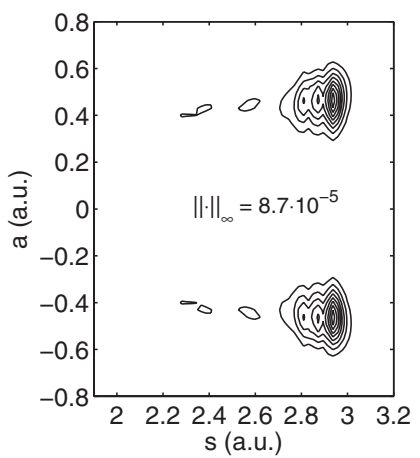

(a)

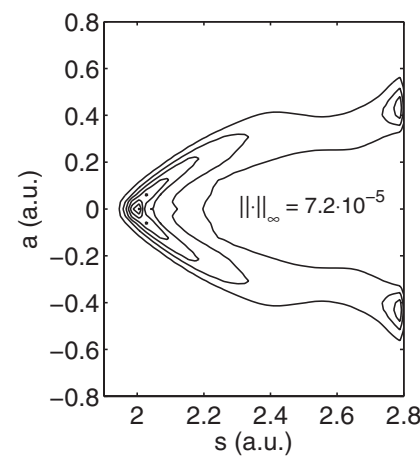

(b)
FIG. 12. Level curves for the pointwise error at time $t=10$ fs for solutions calculated with (a) a control-propagated Hagedorn basis with $32^{2}$ basis functions, and (b) a Fourier basis with $52^{2}$ basis functions. The curve separation is $1.0 \times 10^{-5}$, and the maximum pointwise error is $8.7 \times 10^{-5}$ and $7.2 \times 10^{-5}$ for the Hagedorn and Fourier solutions, respectively. the wave function is negligible by the boundary at the time of comparison, with $248 \times 208$ Fourier basis functions. For the Fourier solution in Figure 12(b), the error is only evaluated in the points which are not inside the absorbing region, i.e. only the part of the domain pictured in the figure. For the Hagedorn solution, the error is evaluated in all points on the reference Fourier grid. The time step was $2.42 \times 10^{-3}$ fs in all simulations.

\section{CONCLUSIONS}

We have presented a method for dynamic adaptation of the Hagedorn basis to the evolving wave function. By matching the support of the basis to the wave function we can achieve smaller error with fewer basis functions. Numerical experiments on one- and two-dimensional molecular systems show how the error can be reduced by orders of magnitude compared to simulation with semiclassical propagation of the basis. Deviation from the semiclassical propagation scheme rids us from the need of knowing the Hessian of the potential, which typically is expensive to calculate. By using a PD controller for the adaptivity we are not dependent on smoothness, or even continuity, of the control target. We can then make use also of crude support estimates for the wave function which come essentially for free. Experiments on a model of IBr illustrates how the control approach can improve accuracy substantially when several electronic states are involved. We also provide a motivation for why a collocation method, with reduced computational complexity, induces little additional error compared to direct exponential integration of the Galerkin formulation. The computation times for the numerical examples in this paper, which were implemented in Matlab and run on a laptop computer, were reduced from hours to minutes by using the collocation method. The computational work does however scale quadratically with the number of basis functions, and the computation times for the presented examples are longer than for the Fourier method. Similarly to other grid methods, the number of basis functions needed scales exponentially with the number of dimensions. Still, moving grid methods like the present provide a promising framework for higher dimensional problems since adaptivity can reduce the required number of basis functions, and thus also the amount of data. For parallel computation of big problems, the amount of data needed is usually a more severe bottleneck than the computational work due to limitations both in cache size and communication throughput. The possible savings are greatest when the wave packet stays localised, but moves over a considerable area. In a fixed grid setting one would have to resolve the entire domain at all times. With a moving grid we only need to resolve the subdomain currently occupied by the wave packet. The savings are considerable already in $1 \mathrm{D}$, and increasingly so in higher dimensions.

This paper describes how our solution method can be applied to problems where the solution consists of a localised wave packet but where quantum phenomena are still important for an accurate description of the dynamics. This is also the class of problems where we expect our method to be the most useful. Such problems arise e.g. from photodissociation. ${ }^{35,36}$ As for other approaches using a 
localised representation, $2,19,37$ additional mechanisms must often be introduced when handling problems with more complex dynamics. Also, some bound state problems are normally best handled by using the standard Fourier representations, as we do for the ground state in the IBr example in Sec. VI. In some applications involving, e.g., tunnelling a wave function may split. For efficient treatment of such cases within our framework one must introduce a methodology for the splitting of the basis, so that different "families" of basis functions follow disconnected parts of the wave function, similarly to what is done in the schemes in Refs. 19 and 37. One would then not have to resolve the unoccupied area between the wave packets. Such splitting of Hagedorn wave packets has been studied previously in a simplified setting. ${ }^{32}$ How to conduct splitting and merging automatically remains an area of future research.

\section{ACKNOWLEDGMENTS}

Discussions with Bengt Carlsson, Vasile Gradinaru, Magnus Gustafsson, Katharina Kormann, Hans Norlander, and Elias Rudberg are gratefully acknowledged.

${ }^{1}$ M. F. Herman and E. Kluk, Chem. Phys. 91, 27 (1984).

${ }^{2}$ D. Kosloff and R. Kosloff, J. Comput. Phys. 52, 35 (1983).

${ }^{3}$ J. C. Light, I. P. Hamilton, and J. V. Lill, J. Chem. Phys. 82, 1400 (1985).

${ }^{4}$ G. A. Hagedorn, Commun. Math. Phys. 71, 77 (1980).

${ }^{5}$ G. A. Hagedorn, Ann. Phys. 269, 77 (1998).

${ }^{6}$ E. Faou, V. Gradinaru, and C. Lubich, SIAM J. Sci. Comput. (USA) 31, 3027 (2009).

${ }^{7}$ S. Adhikari and G. D. Billing, J. Chem. Phys. 113, 1409 (2000).

${ }^{8}$ G. D. Billing and S. Adhikari, Chem. Phys. Lett. 321, 197 (2000).

${ }^{9}$ K. L. Feilberg, G. D. Billing, and M. S. Johnson, J. Phys. Chem. A 105, 11171 (2001).

${ }^{10}$ K. Gustafsson, M. Lundh, and G. Söderlind, BIT 28, 270 (1988).
${ }^{11}$ C. J. Budd, W. Huang, and R. D. Russell, Acta Numerica 18, 111 (2009).

${ }^{12}$ L. R. Pettey and R. E. Wyatt, Chem. Phys. Lett. 424, 443 (2006).

${ }^{13}$ D. Neuhasuer and M. Baer, J. Chem. Phys. 90, 4351 (1989).

${ }^{14}$ J.-P. Berenger, J. Comput. Phys. 114, 185 (1994).

${ }^{15}$ J. P. Boyd, J. Comput. Phys. 54, 382 (1984).

${ }^{16}$ J. P. Boyd, Chebyshev and Fourier Spectral Methods, 2nd ed. (Dover, New York, 2001).

${ }^{17}$ T. Tang, SIAM J. Sci. Comput. (USA) 14, 594 (1993).

${ }^{18}$ H. Ma, W. Sun, and T. Tang, SIAM (Soc. Ind. Appl. Math.) J. Numer. Anal. 43, 58 (2005).

${ }^{19}$ M. Ben-Nun, J. Quenneville, and T. J. Martínez, J. Phys. Chem. A 104, 5161 (2000).

${ }^{20}$ B. Fornberg, A Practical Guide to Pseudospectral Methods (Cambridge University Press, 1996).

${ }^{21}$ E. J. Heller, J. Chem. Phys. 75, 2923 (1981).

${ }^{22}$ G. H. Golub and J. H. Welsch, Math. Comput. 23, 221 (1969).

${ }^{23}$ G. Franklin, J. D. Powell, and A. Emami-Naeini, Feedback Control of Dynamic Systems, 5th ed. (Prentice-Hall, Upper Saddle River, 2006).

${ }^{24}$ H. Liu, O. Runborg, and N. M. Tanushev, "Error estimates for Gaussian beam superpositions," Math. Comput. (to be published).

${ }^{25} \mathrm{~K}$. Kormann and A. Nissen, in Proceedings of the 2009 ENUMATH Conference, Uppsala, 2009, edited by G. Kreiss, P. Lötstedt, A. Målqvist, and M. Neytcheva (Springer-Verlag, Berlin, 2010), pp. 523-531.

${ }^{26}$ H. Guo, J. Chem. Phys. 99, 1685 (1993).

${ }^{27}$ R. Bourquin, V. Gradinaru, and G. A. Hagedorn, J. Math. Chem. 50, 602 (2012).

${ }^{28}$ G. Strang, SIAM (Soc. Ind. Appl. Math.) J. Numer. Anal. 5, 506 (1968).

${ }^{29}$ M. D. Feit, J. A. Fleck, and A. Steiger, J. Comput. Phys. 47, 412 (1982).

${ }^{30}$ W. Magnus, Commun. Pure Appl. Math. 7, 649 (1954).

${ }^{31}$ K. Kormann, S. Holmgren, and H. O. Karlsson, J. Chem. Phys. 128, 184101 (2008).

${ }^{32}$ V. Gradinaru, G. A. Hagedorn, and A. Joye, J. Chem. Phys. 132, 184108 (2010).

${ }^{33}$ K. C. Kulander and J. C. Light, J. Chem. Phys. 73, 4337 (1980).

${ }^{34}$ K. C. Kulander, C. Cerjan, and A. E. Orel, J. Chem. Phys. 94, 2571 (1991).

${ }^{35}$ E. A. Coronado, V. S. Batista, and W. H. Miller, J. Chem. Phys. 112, 5566 (2000).

${ }^{36}$ D. V. Shalashilin, M. S. Child, and A. Kirrander, Chem. Phys. 347, 257 (2008).

${ }^{37}$ Y. Wu and V. S. Batista, J. Chem. Phys. 118, 6720 (2003). 